\title{
Giant photodetector arrays underpin Nobel success
}

\author{
A total of more than 20,000 bespoke photomultiplier tube (PMT) detectors were involved in the \\ experiments behind this year's Nobel Prize in Physics.
}

The 2015 prize, announced on 6 October, was shared between Takaaki Kajita from the University of Tokyo in Japan and Arthur McDonald from Queen's University in Kingston, Canada for "the discovery of neutrino oscillations, which shows that neutrinos have mass."

Kajita is a member of the

Super-Kamiokande (Super-K) collaboration - a neutrino detection experiment that takes place in a former mine in the mountainous area of Gifu in Japan and involves a huge (39.3 m diameter, $41.4 \mathrm{~m}$ tall) subterranean stainless-steel cylinder filled with 50,000 tons of ultrapure water and lined with around 13,000 PMTs (pictured). On the other side of the Pacific Ocean, in Sudbury, Canada, McDonald is the leader of a smaller neutrino detector (12 $\mathrm{m}$ diameter) called the Sudbury Neutrino Observatory (SNO), consisting of an acrylic sphere $2 \mathrm{~km}$ underground, filled with heavy water and surrounded with 9,600 PMTs.

The concept of both experiments is to capture weak Cherenkov radiation, which represents the optical signature of neutrino interactions with matter. As neutrinos have no electrical charge and only very weakly

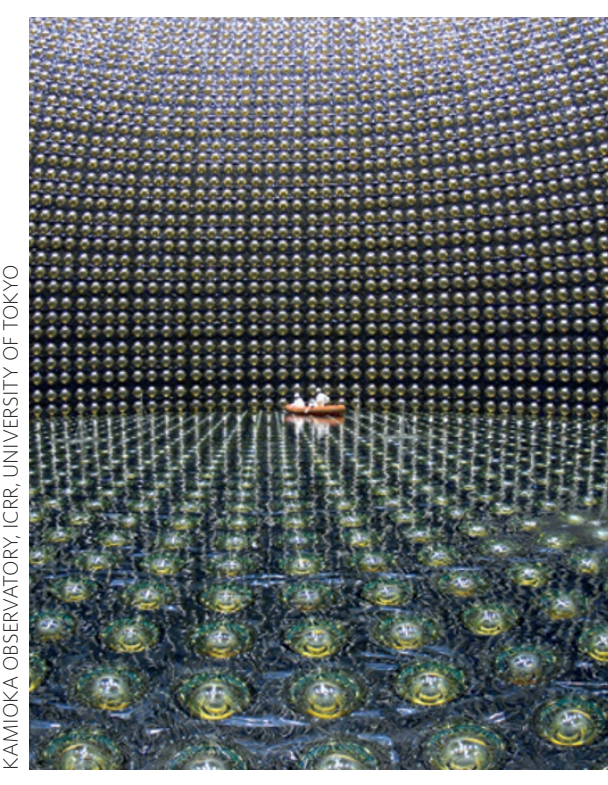

interact, this is not an easy task and both experiments require not only sensitive, large PMTs (Super-K's are $50 \mathrm{~cm}$ across) but also need to be conducted deep underground to avoid contamination from cosmic rays, which would also generate events.
Construction of the Super-K experiment started in 1991 and observations commenced five years later in 1996. In 1998, it provided experimental data that indicated that neutrinos were able to switch flavours or 'oscillate' between their tau, muon and electron types. Experiments at SNO in 2001 provided further evidence and corroborated the phenomenon.

The discovery of these neutrino oscillations was important because it solved a long-standing conundrum called the solar neutrino problem, whereby a far smaller number than predicted of electron neutrinos generated in the Sun were being detected on Earth. Significantly, it also presented evidence for neutrinos having a small mass, rather than being massless as the standard model of particle physics suggests. Interestingly, the Super-K detector suffered a serious, shattering set-back in 2001 when an implosion accident during the refilling of the tank with water damaged more than half of its PMTs. Following reconstruction, experiments recommenced in 2002 using strengthened PMTs featuring fibre-reinforced plastic casings and acrylic front windows.

\section{A solar checklist}

\section{Authors of papers related to solar-cell research will now be asked to complete a checklist of submitted information to aid the reproducibility and transparency of results.}

Following discussion with experts in the photovoltaic community and the publication of several articles on the topic of mischaracterization of solar cell efficiency ${ }^{1-4}$, Nature Photonics (along with other physical science research journals published by Nature Publishing Group) is introducing a checklist for papers submitted on the topic. The idea is that authors submitting results in the area will be asked to download and complete the checklist (http://www.nature. com/authors/policies/solarchecklist.pdf) prior to the manuscript undergoing peer review to enhance the transparency and reproducibility of the work. The checklist will be shown to reviewers as part of the peerreview process, but it will not be published.
The checklist is applicable to all material types and will be applied to all papers where it is deemed to be relevant. The aim is to guide authors in providing salient information and detailing procedures that were undertaken during their research. It encompasses topics such as detailing the size and stability of cells, the number of samples tested, the use of a mask and the illumination conditions during characterization, and whether an accredited third party has certified the cell efficiency.

The introduction of checklists is not new at Nature Publishing Group, as our journals in the life sciences have for some time made use of a similar form to provide important information about issues related to data reporting, statistics and ethics. We believe passionately in supporting communities to encourage best practices and transparency when reporting data and we hope that this checklist will be viewed as being valuable.

We would like to thank all the experts who have provided us with feedback and suggestions during the preparation of the checklist. We are confident that it will become a useful tool to help ensure the high standards that research in the photovoltaics community deserves.

\footnotetext{
References

1. Nature Photon. 8, 665 (2014)

2. Zimmermann, E. et al. Nature Photon. 8, 669-672 (2014)

3. Snaith, H. J. Nature Photon. 6, 337-340 (2012).

4. Timmreck, R. et al. Nature Photon. 9, 478-479 (2015).
} 